\title{
AGENDA DE GÉNERO Y DE PRIMERA INFANCIA: DIÁLOGO SOBRE LOS RETOS QUE PLANTEA EL NUEVO CURRÍCULUM DE EDUCACIÓN PARVULARIA
}

\author{
María Isabel Díaz Pérez.; Bernarda Pérez Carrillo
}

\begin{abstract}
RESUMEN/ ABSTRACT
Este artículo describe el contexto de la agenda pública impulsada en años recientes desde el Estado para abordar el género y la primera infancia. Se identifican, además, iniciativas relevantes que han buscado instalar esta agenda en la implementación de las política de educación y género en Chile. En este marco, dos ex-Subsecretarias de Ministerios clave en el ámbito de la educación y la igualdad de género, cada una desde su perspectiva y considerando sus experiencias vividas desde el rol que tuvieron, dialogan sobre aspectos relevantes referidos a logros, dificultades y desafíos que enfrentan el Estado y la sociedad chilena respecto de la profundización de las transformaciones de la educación para la igualdad de género. Se hace un énfasis particular en la dimensión curricular y en el nivel de Educación Parvularia.
\end{abstract}

Palabras clave: Educación Parvularia; igualdad de género; políticas educativas; currículum

This article describes the context of the public agenda promoted in recent years by the State to address gender and early childhood. It also identifies relevant initiatives that have sought to install this agenda in education and gender policy in Chile. In this framework, two former Undersecretaries of key Ministries in the field of education and gender equality, each from their perspective and considering their experiences from the role they played, discuss relevant aspects related to achievements, difficulties and challenges that the State and Chilean society face regarding the deepening of the transformations of education for gender equality. Particular emphasis is placed on the curricular dimension and the level of Early Chilhood Education.

Keywords: Early Chilhood Education; gender equality; educational policies; curricula 


\section{INTRODUCCIÓN}

Chile se encuentra en un momento histórico de gran intensidad, en el que se ha buscado desarrollar transformaciones profundas en sus bases culturales, sociales, económicas y políticas. En ese camino, como se señala en reportes de PNUD (2017) y OCDE (2017), es innegable que en los últimos años, a través de políticas públicas orientadas a un desarrollo más inclusivo, ha habido avances significativos en la disminución de las brechas de desigualdad.

De acuerdo con lo que afirma la Cuenta Pública del Ministerio de Educación (2017), los esfuerzos del Estado se han orientado no solo a la generación de condiciones de acceso, sino también a establecer modificaciones estructurales para enfocar el futuro del país de un modo distinto, de manera tal que todos y todas puedan acceder a su realización personal y colectiva con pleno respeto a sus derechos, hoy en día, ampliamente reconocidos en la sociedad.

Sobre la base de estas expectativas, a nivel de política pública se activaron Agendas de Género y de Primera Infancia, con iniciativas que se reflejaron en las programaciones de la Subsecretaría de Educación Parvularia (2017) y el Ministerio de la Mujer y Equidad de Género (2018), las que fueron respaldadas por instrumentos legales y acuerdos internacionales.

El siguiente listado identifica los documentos que estuvieron considerados en la definición de las mencionadas Agendas: Convención sobre los Derechos del Niño de 1989; Declaración Mundial sobre Educación para todos de Jomtien, 1990; Marco de Acción de Dakar, 2000; Marco de Acción de Moscú, 2010; Programa de Acción Mundial para el Desarrollo Sostenible de UNESCO, 2014; Declaración sobre la Eliminación de la Violencia contra la Mujer, ONU, 1993; Estrategia de Montevideo para la Implementación de la Agenda Regional de Género en el Marco del Desarrollo Sostenible hacia 2030, CEPAL, 2015; Políticas Públicas e Institucionalidad de Género en América latina, CEPAL, 2012;

Foro Económico Mundial, Global Gender Gap Report, 2011; Plan de Igualdad para Hombres y Mujeres 2018 - 2030, Ministerio de la Mujer y Equidad de Género, 2017. 
En este contexto, en el transcurso del segundo gobierno de la presidenta Michelle Bachelet, se fueron concretando medidas enfocadas a asegurar las condiciones para establecer una ruta sin retrocesos hacia la equidad de género y hacia la equidad educacional desde la primera infancia. Dentro de estas medidas figuran la creación de organismos especializados para fortalecer las capacidades del Estado, la generación de marcos regulatorios para asegurar derechos, y la elaboración de dispositivos y mecanismos para responder a los requerimientos de mayor equidad.

Entre las medidas relativas a la creación de organismos especializados, se encuentra la ley 20.820 que crea el Ministerio de la Mujer y la Equidad de Género; y la ley 20.835 que crea la Subsecretaría de Educación Parvularia, la Intendencia de Educación Parvularia y modifica diversos cuerpos legales. Por su parte, respecto de los marcos regulatorios, destaca la ley 20.840 de Reforma electoral binominal que crea un sistema electoral proporcional con criterio de paridad. En cuanto a iniciativas orientadas a la equidad, destaca la promulgación de la ley 20.915 y la ley 20.900 orientadas a la modernización de partidos políticos y al financiamiento públicos de los mismos, con criterio de paridad de género; la ley 20.845 de la Reforma Educacional que establece que el sistema educativo propenderá a eliminar todas las formas de discriminación arbitraria que impidan el aprendizaje y la participación de los y las estudiantes; y la Circular $N^{0} 0768$ de la Superintendencia de Educación, sobre derechos de niñas, niños y estudiantes trans en el ámbito educativo.

Políticas como las mencionadas se han inspirado de manera explícita en la "perspectiva de derechos" (Sepúlveda, 2014), la que plantea grandes desafíos en cuanto a sus exigencias en términos de la coherencia que debe existir entre los principios que subyacen en el diseño de las políticas y los instrumentos o mecanismos que les dan funcionamiento. Esta perspectiva demanda que se resguarde su consistencia, considerando la multiplicidad de factores que inciden en la efectividad de la puesta en práctica de las políticas. Como lo señala Díaz (2017), “el trabajo intersectorial, dado el carácter integral y sistémico, exige que el Estado asuma con especial protagonismo la responsabilidad de generar mecanismos que optimicen la coordinación vertical y horizontal” (p.9). 
Es importante observar que las transformaciones políticas descritas se implementaron en un contexto de cambios socioculturales protagonizados por diferentes actores y organismos del Estado y la sociedad civil expresados en iniciativas sociales, nacionales e internacionales como, por ejemplo, el movimiento social "Me too" en países europeos y "Ni una Menos" en América Latina. Iniciativas como estas, entre muchas otras, han buscado visibilizar la violencia contra las mujeres, estableciendo vínculos con la necesidad de abordar una educación no sexista. Asimismo, es necesario mencionar en este ámbito, el creciente auge de la incidencia del movimiento feminista, presente en universidades y establecimientos educacionales en Chile.

El diseño de las Agendas de Igualdad de Género e Igualdad Educativa para la primera infancia requirió de la existencia de marcos conceptuales, validaciones internacionales y diversos dispositivos de coordinación. Sobre la base de estos procesos, se fueron creando significados compartidos respecto de las políticas introducidas, lo que se esperaba contribuyera positivamente a su puesta en práctica y a fortalecer su continuidad más allá de los cambios de gobierno. En particular, en este caso, uno de los principales significados que se buscó fuera compartido fue el de la igualdad de género, entendiendo que lo que se quería, a partir de las Agendas, era igualar derechos, responsabilidades, y oportunidades de mujeres y hombres, y de las niñas y de los niños (UNESCO, 2011).

Las agendas mencionadas incluyeron un conjunto de políticas y normas, las que aportaron a dar operatividad a sus enfoques y visiones. Uno de los procesos clave que canalizó la implementación de estas agendas fue el nuevo Currículum oficial para la Educación Parvularia recientemente aprobado por el Consejo Nacional de Educación (2018). El currículum en este nivel de enseñanza, al igual que en otros niveles educativos, condensa el proyecto de sociedad que se busca construir, enunciando fundamentos, orientaciones valóricas, principios pedagógicos, fines educativos, objetivos de aprendizaje y orientaciones para la enseñanza. 


\section{DIÁLOGO ENTRE EX-SUBSECRETARÍAS: EXPERIENCIAS VIVIDAS Y DESAFÍOS PARA LA IGUALDAD DE GÉNERO Y LA IGUALDAD EDUCATIVA}

¿Cómo se abordaron los desafíos de las Agendas de Igualdad de Género y de Igualdad Educativa desde el relato y visión de protagonistas de un gobierno saliente que se desempeñaban en instituciones clave para la vinculación entre la educación y la igualdad de género?

Para responder esta interrogante, se realizó un diálogo entre dos exautoridades, ambas ex-Subsecretarias de distintos Ministerios, a quienes correspondió asumir tareas fundacionales en el ámbito de estas políticas públicas: María Isabel Díaz (ex-Subsecretaria de Educación Parvularia) y Bernarda Pérez (ex-Subsecretaria de la Mujer). En este diálogo tuvo lugar un intercambio entre ambas especialistas acerca de los desafíos involucrados en la implementación de un nuevo currículum, en el marco de las agendas orientadas a promover la igualdad de género y la igualdad educativa. La realización de este intercambio buscó dar prioridad al análisis en "primera persona" de las temáticas enunciadas anteriormente. Para ello, ambas exautoridades diseñaron una pauta para entrevistarse mutuamente, de manera de relevar su visión subjetiva respecto de los procesos que tuvieron que liderar.

El diálogo se estructuró en dos áreas temáticas: 1) Análisis de las políticas en que las entrevistadas tuvieron participación, donde se abordó la implementación y el vínculo entre las Agendas de Igualdad de Género e Igualdad Educativa y 2) Aprendizajes desarrollados e identificación de desafíos relevantes para el desarrollo de una educación para la igualdad de género.

\section{EXPERIENCIAS DE IMPLEMENTACIÓN}

María Isabel Díaz: Desde la experiencia que ha significado participar en el diseño de políticas públicas desde el Ministerio de la Mujer y Equidad de Género, y el vínculo que estas políticas han establecido con el sistema educativo, ¿cómo debiera abordarse el desafío 
de desarrollar una educación no sexista desde el currículum y la formación inicial y continua de los docentes?

Bernarda Pérez: El contexto actual demanda una educación no sexista. Por lo tanto, preguntarnos acerca de cómo se responde a esta demanda en la práctica pedagógica es de toda pertinencia. Lo anterior implica el desafío constante para las instituciones formadoras de docentes de volver a mirar sus mallas curriculares y sus prácticas educativas.

Debemos tener presente que la diferenciación sexista se ha sostenido en acentuar la diferencia en una escala jerárquica que ha terminado por sostener el argumento de que las mujeres son inferiores y en esto la educación en todos sus sistemas ha contribuido. ¿Puede ahora la educación contribuir a la erradicación del sexismo? Yo creo que sí, sin embargo, es necesario el reconocimiento de la discriminación, de la desigualdad y de la segregación como problemas educativos. Por eso fue muy relevante trabajar en conjunto con la Subsecretaría de Educación Parvularia para la incorporación del enfoque de género en el currículum educativo. No obstante, según mi parecer, sigue habiendo un gran desafío para la formación docente y continua en relación con la educación no sexista.

María Isabel Díaz: ¿Qué evaluación harías de la coordinación que existió entre ambos Ministerios para abordar estos desafíos, durante el período que te desempeñaste como Subsecretaria?

Bernarda Pérez: Hubo coordinación con algunas instancias formadoras para la incorporación de estos temas, sobre todo con las dos nuevas universidades que se formaron durante el período de gobierno en que ejercí como Subsecretaria. Allí, las Secretarías Regionales Ministeriales, así como las Direcciones Regionales de Educación y de la Mujer, estuvieron brindando orientaciones para incorporar esta mirada tanto en mallas curriculares como en los estatutos de estas. No obstante, no todo lo que se planteaba como necesario de implementar quedó instalado como hubiéramos esperado. Ahí tienes un claro ejemplo de que el cambio cultural implica involucrarse desde diferentes ámbitos en los desafíos orientados hacia la igualdad de género. Es relevante tener presente que los pasos en materia curricular han sido fundamentales, pero aún hay mucho que hacer, a nivel 
intergubernamental, para instalar sosteniblemente la Agenda de Género en el sistema educativo.

Bernarda Pérez: Anteriormente, hemos enunciado la relevancia del currículum en una política orientada a la igualdad de género. A tu juicio, ¿cómo se visibiliza o explicita la perspectiva de género en el nuevo currículum para la educación parvularia y qué desafíos se presentan en la práctica pedagógica?

María Isabel Díaz: La incorporación de la dimensión de género en el currículum para la primera infancia se establece desde un enfoque inclusivo, el que se presenta dentro de los fundamentos que orientan la propuesta. Allí, se hace referencia explícita a marcos normativos y tratados internacionales ratificados por el país, por ejemplo, la Convención de los Derechos del Niño. En cierta medida es una declaración de principios que orienta el trabajo educativo.

Con el propósito de concretar estos principios a nivel de logros de aprendizaje se buscó incorporar también el enfoque inclusivo en los diferentes "Núcleos de aprendizaje" que son ámbitos de acción para la experiencia educativa. Lo que se buscaba alcanzar era que los niños y las niñas incorporaran la idea de una sociedad social y culturalmente diversa en la que se valora a todos y a todas por igual.

Tú te hacías la pregunta sobre si la educación puede contribuir a la erradicación del sexismo y yo coincido contigo en que esto es posible, pero que, para ello, se requieren acciones en diversos planos. Por ejemplo, para explicitar la incorporación de la dimensión de género en forma concreta, en la presentación del Núcleo de Aprendizaje sobre Exploración del Entorno Natural, se describe que es necesario considerar la perspectiva de género en los roles y responsabilidades que asumen los integrantes al realizar trabajos grupales. Estos son mensajes curriculares que deben adquirir visibilidad en las aulas. Un segundo ejemplo que podría destacar ocurre en materia de Logros de Aprendizaje definidos en las Bases Curriculares, en el Nivel de Transición (entre 4 y 6 años). Allí se plantea un objetivo con el que se espera potenciar la comprensión y apreciación de la dimensión social y cultural desde una mirada amplia e inclusiva. Ese objetivo es: conocer sobre la vida de algunas mujeres y hombres que han realizado, en el pasado y en el presente, aportes 
diversos en su comunidad, en su país y en el mundo, a través de relatos o con apoyo de TICs. En este caso, se menciona en forma explícita que deben incorporarse mujeres y hombres, pero la complejidad de este objetivo implica contar con la información, buscarla, seleccionarla, relevarla. Considerando lo anterior, la implementación curricular es definitivamente un gran desafío, donde se requieren herramientas didácticas y de acompañamiento.

Hace unos días atrás tuve una jornada con docentes y les pregunté, a propósito de ese objetivo, por mujeres que se habían destacado en su región, en diferentes ámbitos. Ellos y ellas manifestaron, en forma muy franca, que no tenían suficiente repertorio. Solo conocían destacadas figuras, como Gabriela Mistral. Esos son los retos en el trabajo pedagógico, puesto que no solo se trata de definir un Objetivo de Aprendizaje. Otro desafío de gran envergadura es cómo visibilizar dicho objetivo desde la didáctica.

Me hace mucho sentido lo que planteaste en la reflexión anterior sobre la necesidad de incorporar estas temáticas en la formación inicial y continua. Sin embargo, esta incorporación no es sencilla puesto que requiere de un riguroso repertorio metodológico para tomar conciencia de las prácticas discriminatorias.

\section{DESAFÍOS PARA LA PROFUNDIZACIÓN DE LA VISIÓN DE IGUALDAD DE GÉNERO EN EL CAMPO EDUCATIVO}

María Isabel Díaz: El desarrollo de comunidades educativas inclusivas involucra y compromete directamente a las familias, las que transmiten en su cotidianidad creencias, visiones y roles asociados al género. Desde tu perspectiva, ¿cómo ha sido la experiencia de abordar políticas y estrategias dirigidas a afectar estos espacios íntimos de formación y qué desafíos habría que tener en cuenta para su adecuado despliegue?

Bernarda Pérez: La principal dificultad es que la organización de nuestra sociedad se basa en la división sexual del trabajo. Desde esa estructura se definen espacios prioritarios para mujeres y hombres, asignando a los varones el rol de proveedores y a las mujeres el rol de cuidado y tareas domésticas. Como resultado de esta situación, las mujeres enfrentan 
distintos tipos de barreras que les impiden el acceso, el desarrollo y la permanencia, por ejemplo, en el trabajo remunerado en igualdad de condiciones.

Nuestra agenda se sustentó en la perspectiva que indica que es el medio familiar el espacio en que se asientan las bases de los principios igualitarios, que luego se trasladarán al mundo sociolaboral y a la futura familia. La colaboración entre los miembros de la familia ofrece pautas para fomentar el cambio de los patrones de distribución, contribución y acuerdo de los miembros de la familia en la organización del trabajo familiar cotidiano: a eso le llamamos corresponsabilidad. Desde el Ministerio de la Mujer y Equidad de Género nos propusimos diferentes acciones, algunas de sensibilización, a través de campañas de difusión, como por ejemplo "Compartamos la pega"1; y otras más estructurales, como cuerpos legales que permitieran ir garantizando mayor equidad en el trabajo de cuidado entre hombres y mujeres.

Las cifras nos dicen que en Chile existe un promedio de 1.300 .000 mujeres que no trabaja fuera del hogar por razones familiares, es decir, por estar cuidando a sus hijos/as, a un familiar enfermo/a, a un abuelo/a, a una persona dependiente. Conciliar el trabajo no remunerado con el trabajo remunerado es un tema complejo para las mujeres, toda vez que es aún muy fuerte la representación social de que la mujer es la principal o única responsable del trabajo doméstico y de cuidado de otros.

Para ir enfrentando estas desigualdades, se impulsaron acciones relevantes en conjunto con otras carteras, tales como nuevas salas cunas y aulas nivel medio de jardín infantil que implicaron nuevos cupos para niños/niñas de 0 a 4 años. Por otra parte, se aprobaron importantes leyes que aportan al objetivo de la corresponsabilidad: la ley 20.761 que extiende a los padres trabajadores el derecho de alimentar a sus hijos/as; la ley 21.063, de Seguro para el Acompañamiento de los niños y niñas afectados por alguna situación grave de salud, para que puedan ser cuidados por sus padres; la ley 20.891 que perfecciona el permiso posnatal parental y el ejercicio del derecho a la sala cuna para funcionarias y funcionarios públicos, eliminando el tope de remuneración para pago de permiso posnatal

\footnotetext{
1 "Compartamos la Pega" fue una campaña realizada por el Ministerio de la Mujer y Equidad de Género durante los años 2016 y 2017, cuyo objetivo era invitar a las familias a comprometerse con la igualdad y equidad en el trabajo doméstico, con el fin de avanzar en desnaturalizar el rol de las mujeres según el cual han de hacerse cargo de las labores domésticas, crianza y cuidado.
} 
parental y permitiendo que la madre funcionaria pública pueda utilizar la sala cuna del servicio o ministerio del padre del menor, cuando ambos sean funcionarios públicos.

Todas estas medidas apuntan a la corresponsabilidad en las tareas del cuidado de niños y niñas y, como país, debemos seguir avanzando y fortaleciendo lo construido. La corresponsabilidad es necesaria para avanzar en un desarrollo económico inclusivo, equitativo y sustentable. Democratizar las labores de la casa es una necesidad que nuestro país debe resolver en el corto plazo.

Si pensamos en el futuro y los desafíos que se presentan respecto de la igualdad de género y su vínculo con la educación, a mi juicio, la labor más compleja de realizar es sensibilizar a la sociedad en general sobre la necesidad de mover las barreras culturales que impiden este objetivo.

Del mismo modo, debemos avanzar en cambios legislativos y de construcción de sociedad hacia un Sistema Nacional de Cuidado, acción que implica comprometer al Estado y a la empresa privada porque implica recursos. Construir una nueva cultura de sociedad es una tarea a la que todas y todos estamos convocadas/os: el Estado, las empresas, la familia, el jardín infantil, la escuela, la educación superior. Todos/as debemos estar comprometidos con las transformaciones que nuestro país requiere.

Hablemos ahora de las dinámicas de enseñanza, donde finalmente debieran expresarse las grandes políticas que ya han sido mencionadas, pero donde se presentan aún grandes desafíos. Una representación clara de la persistencia de estos desafíos se evidencia en la presencia de estereotipos en la educación. Por ejemplo, las imágenes que se usan en los procesos de enseñanza comunican visiones de hombres y mujeres, representan roles, actitudes o determinados desempeños que pueden adquirir una estructura de estereotipo. Sobre esta forma de transmitir estereotipos sexistas, ¿qué acciones se proyectan como relevantes de realizar para modificar estas expresiones?

María Isabel Díaz: Esta dimensión forma parte de lo que nosotros llamamos currículum oculto, que es aquello que no se explicita, pero que se enseña y/o manifiesta en la práctica cotidiana en las aulas, sin que necesariamente se reflexione sobre ello o se tenga conciencia al respecto. Aprender a descubrir los estereotipos presentes en los procesos educativos 
involucra un proceso de profunda reflexión. Hace años hice una tesis de magister en la que precisamente estudié las imágenes que se exponen en los muros de las aulas y encontré, en toda la muestra de los establecimientos de una comuna, representaciones estereotipadas de niños y niñas. No estamos hablando solo de colores o de análisis cuantitativos en una clase, se trata de la visión cultural que estas imágenes representan. Por ejemplo, en el estudio que estoy describiendo, recuerdo las actitudes de las ilustraciones de las mujeres: coquetas, seductoras, dulces, inactivas. En cambio, los hombres se mostraban activos, arriba de los árboles, corriendo, poderosos e inteligentes. En las entrevistas que realicé en esta investigación, al observar las fotografías de las diversas salas, ninguna de las docentes identificaba o reconocía la presencia de estereotipos, puesto que sus comentarios se centraban en las variables estéticas de las imágenes. Los estereotipos, a fuerza de repetirse, van adquiriendo estructura de maqueta y logran internalizarse en lo más profundo de cada ser y, claro, pueden desestimar cualidades personales, como sucede con los modelos femenino y masculino.

Modificar estas representaciones implica la realización de transformaciones profundas, que afectan a los sujetos participantes de los procesos educativos. Hoy, probablemente por una serie de procesos y factores sociales y culturales, ya se han ido incorporando más elementos para reflexionar sobre temas como el que menciono, lo que es un importante avance para el cambio en las prácticas educativas. Sin embargo, y junto a ello, las acciones formativas dirigidas a profesores y profesoras debieran también aportar a la reflexión sobre la docencia cotidiana, y a la toma de conciencia de cuáles son aquellos repertorios metodológicos que impiden o potencian el desarrollo integral de todas las personas.

En relación con la práctica pedagógica en el aula, entonces, tenemos grandes desafíos que no han sido lo suficientemente abordados, si pensamos en los temas que hemos estado discutiendo. No se trata, por ejemplo, de enviar circulares para normar la pertinencia de las ambientaciones en las aulas. Se requiere reflexionar críticamente sobre el poder cultural que tienen las imágenes en el modelamiento de los comportamientos. Este es solo un caso, pero hay un conjunto importante de visiones y de prácticas respecto de las 
cuales habría que discutir para analizar sus implicancias o efectos respecto de la igualdad de género en la educación.

Producir cambios en este ámbito provoca tensiones y emergen susceptibilidades que debemos tener presente al momento de diseñar estrategias y herramientas. A mi parecer, este tipo de transformaciones solo se han integrado periféricamente en la planificación de los procesos educativos. Me preocupa, además, que el foco se ponga solamente en la figura de la mujer y su visión segregada, porque es necesario comprender que respecto de los hombres también hay visiones fragmentadas de la realidad: por ejemplo, se tiende a excluirlos de las actividades del hogar.

Lo importante es que debemos comprender que los y las docentes somos agentes pedagógicos de género y que la socialización de género se inicia desde el nacimiento.

\section{CONCLUSIONES DEL DIÁLOGO}

Chile ha experimentado, sin lugar a dudas, grandes transformaciones en distintos ámbitos estos últimos años. No obstante, en este diálogo se visibiliza que nuestro país debe seguir avanzando en la realización de acciones concretas que permitan ir cerrando brechas de género, ya que no es posible, en la actualidad, concebir el desarrollo sin que sea imperativo abordar aquellas barreras u obstáculos que dificultan la igualdad en este ámbito.

Dentro de este macropropósito, es clave avanzar hacia un currículum no sexista que se traduzca, a su vez, en prácticas pedagógicas concretas y conscientes. El compromiso de los distintos actores en este ámbito es fundamental para que las definiciones y expectativas se traduzcan en efectos concretos en, por ejemplo, planes de estudio y el aula.

Un trabajo sostenible que se dirija hacia estos logros requiere de un trabajo interinstitucional coordinado en el que el Estado se vincule con otras carteras, organismos y actores, para facilitar la implementación de políticas públicas que favorezcan el derecho a la igualdad entre hombres y mujeres, a través de la remoción de todo tipo de discriminaciones. Asimismo, se necesita que las universidades, escuelas y jardines 
infantiles incorporen mayores esfuerzos en los procesos educativos tendientes a desarrollar una educación no sexista.

\section{REFERENCIAS}

Díaz, M. (2016). Contenido, integralidad y coherencia en las politicas de primera infancia: aportes desde el currículo. Reflexiones en curso sobre cuestiones fundamentales y actuales del currículo, el aprendizaje y la evaluación $\mathrm{N}^{\circ} 7$. UNESCO-OIE. Recuperado de: http://unesdoc.unesco.org/images/0024/002467/246701s.pdf

Ministerio de Educación (2017). Cuenta Pública 2014-2017. Santiago, Chile: Ministerio de Educación de Chile. Recuperado de: http://sitios.mineduc.cl/Cuenta-Publica-2017Libro/files/assets/basic-html/page-1.html

Ministerio de Educación (2018). Bases Curriculares de Educación Parvularia. Recuperado de https://parvularia.mineduc.cl/wpcontent/uploads/sites/34/2018/03/Bases_Curriculares_Ed_Parvularia_2018.pdf

Ministerio de la Mujer y Equidad de Género (2018). Plan Nacional de Igualdad entre Hombres $y \quad$ Mujeres $2018 \quad$ - 2030. Recuperado de https://www.minmujeryeg.cl/ministerio-de-la-mujer-y-la-equidad-de-genero/

OCDE (2017). Evaluaciones de Políticas Nacionales de Educación - Educación en Chile. Santiago, Chile: Fundación SM.

PNUD (2017). Desiguales - Orígenes, cambios y desafios de la brecha social en Chile. Santiago, Chile: PNUD - Uqbar. 
Sepúlveda, M. (2014). De la retórica a la práctica: el enfoque de derechos en la protección social en América Latina. CEPAL. Recuperado de https://repositorio.cepal.org/bitstream/handle/11362/35912/1/S2014131_es.pdf

Subsecretaría de Educación Parvularia (2017). Hoja de ruta: Definiciones de Política para una Educación Parvularia de Calidad. Recuperado de https://parvularia.mineduc.cl/hoja-de-ruta/

UNESCO (2011). Indicadores UNESCO de Cultura para el Desarrollo y la Igualdad de Género. $\quad$ Extraído de 2018 de https://es.unesco.org/creativity/sites/creativity/files/digitallibrary/cdis/Iguldad\%20de\%20genero.pdf 